\title{
O caso de Cariclo: refletindo sobre o papel dos astrônomos na Educação em Astronomia
}

\author{
The case Chariklo: reflecting about the role of astronomers in Astronomy Education
}

\author{
Rodolfo Langhi*1 \\ ${ }^{1}$ Departamento de Física, Universidade Estadual Paulista, Bauru, SP, Brasil
}

Recebido em 05 de Março, 2017. Aceito em 05 de Abril, 2017.

\begin{abstract}
A interação entre astrônomos amadores e profissionais em Astronomia é uma tendência mundial, sendo muitas as descobertas e contribuições dos amadores à comunidade científica. Um exemplo bem-sucedido desta colaboração foi a descoberta do primeiro sistema de anéis ao redor de um corpo menor do Sistema Solar, o centauro denominado Cariclo, que orbita o Sol numa região entre Saturno e Urano. Participaram da equipe internacional de trabalho alguns astrônomos amadores do Brasil cujas observações foram essenciais para esta descoberta. Porém, o caso de Cariclo leva-nos a refletir com mais propriedade sobre a ampliação desta relação entre amadores e profissionais para outra esfera: a escolar. Neste sentido, a atuação conjunta de astrônomos (profissionais e amadores) na educação tem resultado em benefícios à educação de alguns países e à formação de professores com relação a conteúdos e metodologias de ensino de Astronomia. No Brasil, um dos exemplos de destaque é o Ano Internacional da Astronomia, que deixou como legado importantes reflexões sobre o papel dos astrônomos na Educação em Astronomia, o qual também discutimos neste artigo, finalizando com um elenco de desafios e possibilidades para nosso país.

Palavras-chave: Educação em Astronomia; astronomia profissional; astronomia amadora; corpos menores do Sistema Solar.

The interaction with amateur astronomers and professionals is a worldwide trend, with many discoveries and contributions from amateurs to the scientific community. A successful example of this collaboration was the discovery of the first ring system around a smaller body of the Solar System, the Centaur named Chariklo, which orbits the Sun in a region between Saturn and Uranus. Some amateur astronomers from Brazil participated in the international work team whose observations were essential for this discovery. However, Cariclo's case leads us to reflect more closely on the expansion of this relationship of amateurs and professionals to another sphere: the School. In this sense, the union of astronomers (professionals and amateurs) and the education has resulted in benefits to the education of some countries and the teachers formation about contents and methodologies of Astronomy teaching. In Brazil, one of these examples is the International Year of Astronomy, which left as a legacy important reflections about the role of astronomers in Astronomy Education, which we also discussed in this article, ending with a list of challenges and possibilities for our country.
\end{abstract}

Keywords: Astronomy Education; professional astronomer; amateur astronomer; minor planets of Solar System.

\section{Pro-Am: interações entre astrônomos profissionais e amadores}

A interação entre amadores e profissionais em Astronomia é uma tendência mundial, cujo objetivo principal é o amador fornecer dados que, se obtidos de forma sistemática, podem contribuir para trabalhos de pesquisa da comunidade cientifica. Consequentemente, gera-se produção acadêmica em conjunto, mesmo não sendo este o objetivo primário dos amadores, embora seja exigência formal das instituições nas quais trabalham os astrônomos profissionais. Conhecida internacionalmente pela sigla Pro-Am, a colaboração entre a Astronomia Profissional e Amadora já vem ocorrendo desde séculos passados e há ainda um

*Endereço de correspondência: rlanghi@fc.unesp.br grande potencial para continuar fertilmente no futuro [1].

Apesar de a denominação "amador" remeter a uma ideia de atividade de "principiante", ou simplesmente por "amor", muitos destes astrônomos amadores desenvolvem estudos, coordenam trabalhos de observação e publicam resultados em revistas especializadas juntamente com astrônomos profissionais. Em alguns países, organizam-se congressos e workshops entre profissionais e amadores para definirem campanhas de observações colaborativas e projetos do tipo Citizen Science, além de criarem oportunidades aos amadores para trabalharem com redução de dados coletados em alto volume pelos profissionais [1].

Tendo em vista a importância e relevância do trabalho contribuinte dos amadores, concordamos com [2] quando identificam tais astrônomos amadores com a seguinte 
designação: astrônomos amadores semiprofissionais ou simplesmente astrônomos semiprofissionais. Segundo os autores, este termo foi escolhido porque dentro do grupo dos amadores como um todo, há aqueles que consideram a Astronomia apenas um hobby de final de semana. Assim, pode-se dividir os astrônomos em três grandes classes: astrônomos profissionais, astrônomos amadores comprometidos (ou astrônomos semiprofissionais) e astrônomos amadores hobbystas. As descobertas e os estudos realizados pelos amadores incluem: descobertas de supernovas, monitoramento de impactos na Lua, ocultações, atmosferas planetárias, estrelas variáveis, astrometria de NEOs (Near Earth Objects), meteoroides, asteroides, cometas, dentre outros. De fato, vários amadores podem ser considerados profissionais em muitos outros aspectos [1].

De acordo com Langhi e Nardi [3], pelo menos dois fatores contribuem para que haja esta interação: os amadores são em maior número, quando comparado ao dos profissionais; e seus pequenos instrumentos observacionais favorecem determinados tipos de atividades de exploração que, algumas vezes, complementam as dos profissionais.

\section{O caso de Cariclo: um exemplo bem-sucedido da interação Pro-Am}

Um exemplo atual da colaboração Pro-Am envolvendo astrônomos amadores brasileiros foi a descoberta do primeiro sistema de anéis ao redor de um corpo menor do Sistema Solar, denominado Cariclo, que orbita o Sol numa região entre Saturno e Urano. Ao todo, participaram neste trabalho mais de 100 astrônomos profissionais e amadores de vários países, sendo 64 deles envolvidos diretamente na publicação de um artigo final, provenientes de 34 instituições de 12 países diferentes, sendo 13 brasileiros de 7 instituições distintas [4] [5].

Todos os objetos que orbitam em torno do Sol e que são muito pequenos, ou seja, que não possuem massa suficiente para que a sua própria gravidade lhes dê uma forma praticamente esférica, são definidos pela União Astronômica Internacional (IAU) como sendo corpos menores do Sistema Solar. Esta classe inclui atualmente a maioria dos asteroides do Sistema Solar, objetos próximos da Terra, asteroides troianos de Marte e Júpiter, a maioria dos centauros, a maioria dos objetos transnetunianos e os cometas. Informalmente, os termos asteroide e corpo menor são frequentemente usados para indicar objetos de mesma natureza. Os asteroides são pequenos corpos rochosos e/ou metálicos e sua maior parte possui órbitas entre Marte e Júpiter, formando o Cinturão de Asteroides.

Os Centauros diferem dos muito mais numerosos corpos do Cinturão de Asteroides e podem ter vindo da região do Cinturão de Kuiper, um disco fino de corpos de gelo e rocha que se extende de 30 a 55 UA do Sol (1 $\mathrm{UA}=1,50 \times 10^{8} \mathrm{~km}$ ). São pequenos corpos com órbitas instáveis no Sistema Solar exterior, que atravessam as dos planetas gigantes. Como suas órbitas são frequentemente perturbadas por estes planetas, espera-se que permaneçam nelas apenas alguns milhões de anos [6].

Sua designação nominal deriva da mitologia grega, que descreve os centauros como criaturas mistas com cabeça, braços e tronco de ser humano, mas com corpo e pernas de cavalo. Tal como eles, estes corpos menores do Sistema Solar partilham algumas características de duas espécies diferentes, pois podem apresentar aspectos tanto de asteroides quanto de cometas. Quanto ao Cariclo, parece ser mais como um asteroide, não sendo descoberto nele qualquer atividade cometária (na mitologia, Cariclo era filha de Apolo que se casou com o centauro Quíron).

Estudar a região do Cinturão de Kuiper e os objetos transnetunianos (TNO) justifica-se porque tais corpos são considerados como verdadeiros fósseis no Sistema Solar. Os centauros devem ter sofrido pouco com as intempéries interplanetárias e a sua distribuição orbital, dimensões e massa podem estar ligadas à migração planetária na época da formação do Sistema Solar. Assim, ao determinar suas propriedades físicas, como tamanho, densidade, albedo e composição, pode-se compreender a origem do Sistema Solar. Mas, não se previa a existência de anéis em corpos menores e nem se pensava sobre esta possibilidade. Por isso, a descoberta foi uma surpresa para a comunidade científica e amadora [5].

A equipe, composta de astrônomos profissionais e amadores, participa de uma Rede de Ocultações, cujo objetivo é estudar a variação do brilho de uma estrela quando um corpo menor passa em sua frente, sob o ponto de vista de um observador na Terra. Um corpo menor do Sistema Solar com dimensões e distância tal como a dos TNO não pode ser observado com detalhes, mesmo com os maiores telescópios do mundo.

Por exemplo, pensando em um centauro, cujo diâmetro é de $250 \mathrm{~km}$ a uma distância de 13,5 UA (cerca de dois bilhões de $\mathrm{km}$ ), o tamanho aparente deste corpo visualizado por um observador na Terra seria de 0,025 " (segundos de arco), o que equivale a tentar enxergar uma moeda de $\mathrm{R} \$ 1,00$ colocada a $210 \mathrm{~km}$ de distância [5].

Por isso, a solução é simplesmente observar os efeitos da passagem deste corpo na frente de uma estrela, ou seja, basicamente estudar a variação de brilho da estrela. Comparando-se com um eclipse, é como se o objeto formasse uma "sombra" sobre a Terra durante a ocultação. Em se tratando de um corpo pequeno colocado a uma grande distância, assume-se que o tamanho desta "sombra" é igual ao tamanho do objeto. Se houver colaboradores suficientes localizados em diferentes regiões e latitudes da superfície da Terra, torna-se possível determinar com relativa precisão suas diversas características e parâmetros orbitais ao acompanhar a ocultação da estrela pelo corpo. Isto justifica a importância de ampla participação dos astrônomos amadores com seus telescópios (a figura 1 ilustra a união das observações entre profissionais e amadores para o caso Cariclo).

Cariclo é o maior centauro conhecido, com raio de 125 $\mathrm{km}$, orbitando uma região entre Saturno e Urano com 


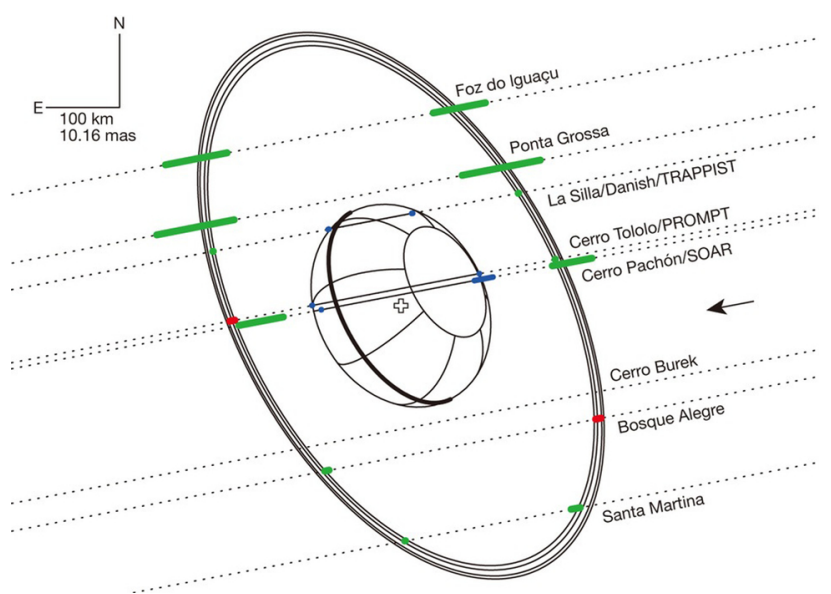

Figura 1: Modelo da geometria dos anéis de Cariclo. As linhas pontilhadas são as trajetórias da mesma estrela em relação a Cariclo no plano do céu, observadas de diferentes estações em Terra. Devido ao efeito de paralaxe, cada observador observa a mesma estrela cruzar Cariclo com trajetórias em diferentes posições. Os segmentos verdes indicam a localização do anel interno conforme observados das estações em Terra. Os segmentos azuis são as cordas de Cariclo observadas das estações em Terra. Fonte: [4].

semieixo maior orbital de 15,8 UA. Foi descoberto em 15 de fevereiro de 1997 por James V. Scotti, da Universidade do Arizona. Sua designação oficial do Minor Planet Center (MPC), da União Astronômica Internacional (IAU), é 10199 Chariklo. O Minor Planet Center (Centro de Planetas Menores) da IAU é o centro que coordena a detecção de pequenos corpos no Sistema Solar. Os nomes dados a estes objetos são constituídos por duas partes: um número e um nome [6].

Durante a madrugada do dia 3 de junho de 2013, o grupo coletou dados para estudar a curva de luz da estrela UCAC4248-108672 de magnitude 12,4 ocultada por Cariclo. A diminuição do brilho da estrela já era esperada devido à passagem de Cariclo na frente dela e seu estudo detalhado forneceria informações que enriqueceriam os modelos já existentes.

As observações deste evento foram realizadas pelos seguintes observatórios profissionais e amadores: telescópios TRAPPIST e dinamarquês de 1,5 metros de diâmetro, situados no Observatório de La Silla do ESO (Chile), cuja curva de luz está apresentada na figura 2; Observatório da Universidad Católica (UCO) Santa Martina operado pela Pontifícia Universidad Católica de Chile (PUC); telescópios PROMPT, que pertencem e são operados pela Universidade da Carolina do Norte em Chapel Hill; Observatório Pico dos Dias do Laboratório National de Astrofísica (OPD/LNA), no Brasil; telescópio Southern Astrophysical Research (SOAR); telescópio Caisey Harlingten's 20-inch Planewave, que faz parte do Searchlight Observatory Network; telescópio de R. Sandness das Explorações Celestes de San Pedro de Atacama; Observatório da Universidade Estadual de Ponta

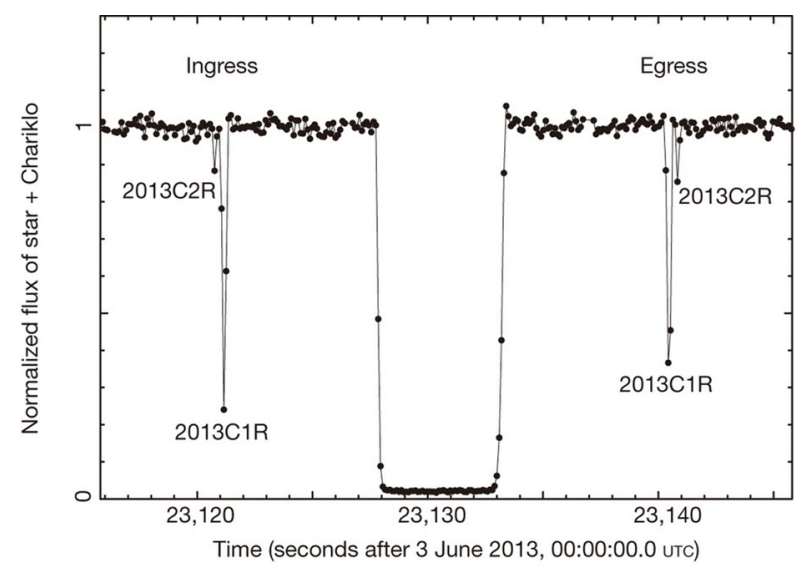

Figura 2: Curva de luz da estrela ocultada por Cariclo, obtida a uma taxa de quase $10 \mathrm{~Hz}$, pelo Telescópio Dinamarquês (La Silla, Chile), com 1,54 $\mathrm{m}$ de abertura. Os dois anéis de Cariclo são evidenciados antes e depois da queda de luz principal. Fonte: [4].

Grossa; Observatorio Astronomico Los Molinos (OALM), Uruguai; Observatorio Astronomico, Estacion Astrofisica de Bosque Alegre, Universidad Nacional de Cordoba, Argentina; Observatório do Polo Astronômico Casimiro Montenegro Filho (Fundação Parque Tecnológico Itaipu, PR) e Observatorio El Catalejo, Santa Rosa, La Pampa, Argentina [6].

Focando nossa atenção em apenas um destes estabelecimentos, encontramos o caso do Polo Astronômico Casimiro Montenegro Filho, em Foz do Iguaçu, PR, cujo observatório em nível amador possui uma cúpula de 6 $\mathrm{m}$ de diâmetro equipado com um telescópio SchmidtCassegrain de 11" (280 mm) e uma câmera CCD SBIG ST-7XME, usando o software CCDSoft. Este observatório é cadastrado no Minor Planet Center (MPC), da International Astronomical Union (IAU), sob o código X57. O controle do horário das observações foi feito por meio de um GPS acoplado ao computador que operava os equipamentos. Naquela noite, foram registradas 150 imagens por um docente da UNIOESTE (Universidade Estadual do Oeste do Paraná) e um dos monitores atendentes deste Polo Astronômico, cujos nomes fazem parte dos 64 autores do artigo resultante, publicado em 26 de março de 2014 [4].

Após as análises das curvas de luz obtidas também por outros observatórios, constataram-se diminuições adicionais da intensidade da luz recebida da estrela durante a passagem de Cariclo, além do decréscimo esperado devido à passagem do centauro em frente à estrela ocultada. Tais quedas do fluxo luminoso antes do ingresso e após o egresso do centauro indicaram a existência de dois anéis, um com raio orbital de $391 \mathrm{~km}$ e largura de $7 \mathrm{~km}$, outro com raio orbital de $405 \mathrm{~km}$ e largura de $3 \mathrm{~km}$, separados por um espaço de $9 \mathrm{~km}$ [6].

Os anéis de Cariclo são formados por fragmentos que contêm, em parte, gelo de água e até esta descoberta, anéis haviam sido identificados apenas nos planetas gi- 
gantes (Júpiter, Saturno, Urano e Netuno). Eles são mais brilhantes que os anéis de Urano e mais escuros que o anel A de Saturno. É provável que existam satélites pastores com tamanho da ordem de $1 \mathrm{~km}$ atuando no confinamento dos anéis de Cariclo, ou então os anéis formaram-se recentemente, o que explicaria este espaçamento entre eles.

Dentre as possíveis causas da existência dos anéis estão: a) um impacto escavou material congelado das camadas exteriores de Cariclo, destruindo um satélite preexistente ou foi fragmentado durante o impacto; b) houve uma ruptura rotacional do corpo principal ou ejeção de material por meio de atividade similar a dos cometas; c) ocorreu uma colisão de dois satélites preexistentes, que se fragmentaram; d) aconteceu uma destruição de um satélite com órbita retrógrada por forças de maré, ao migrar para o interior.

\section{Pro-Am-Es: ampliando a colaboração Pro-Am ao refletir sobre o papel dos astrônomos na Educação em Astronomia}

O caso de Cariclo nos leva a refletir com mais propriedade sobre a relação Pro-Am já existente há alguns séculos. Nota-se a naturalidade com que a Astronomia permite a colaboração entre profissionais e amadores, o que não se verifica com facilidade em outras áreas do conhecimento (não existem "físicos amadores", por exemplo).

Contudo, esta relação pode ser muito mais produtiva em relação ao que já se apresenta, ampliando-se o círculo de envolvimento da colaboração Pro-Am. Levando em conta as sugestões de [3], esta ampliação atingiria a esfera escolar e, neste sentido, a conhecida sigla passaria a ser designada como Pro-Am-Es. Em contrapartida, a garantia de manutenção das aproximações Pro-Am será fortalecida principalmente se houver investimentos de esforços na base: Divulgação e Educação.

Voltando ao exemplo do Polo Astronômico Casimiro Montenegro Filho, o qual oferece um programa de formação de professores sobre conteúdos e metodologias de ensino de Astronomia, registra-se que o caso de Cariclo foi inserido por alguns destes professores no ensino de Ciências das escolas do estado do Paraná, após este espaço não formal efetuar a transposição didática deste conhecimento científico. A formação destes professores também tem base em colaborações de astrônomos profissionais e amadores locais, regionais, nacionais e internacionais. Por exemplo, este ambiente de ensino é certificado pela IAU por meio da Network for Astronomy School Education (NASE), atuando desde 2009 nas linhas de popularização da Astronomia, ensino não formal e formação de professores.

Internacionalmente, a preocupação entre as relações Pro-Am-Es começa a se fortalecer a partir de 1967, durante uma das reuniões da IAU na Tchecoslováquia. A
IAU possui a Comissão 46 (Ensino), cuja finalidade é desenvolver, aperfeiçoar e disseminar informações concernentes à Educação em Astronomia em todos os níveis do mundo mediante vários projetos. Nesta ocasião, a Comissão 46 da IAU decidiu preparar uma lista de todos materiais disponíveis voltados para a Educação em Astronomia e em 1970 surgiu a primeira publicação intitulada: "Astronomy Educational Material", onde o Brasil surge, pela primeira vez, no período 1985-1988, com dez trabalhos ali publicados [7]. Em julho de 1988, durante a reunião da IAU nos Estados Unidos, 162 astrônomos de 31 países objetivaram discutir o tópico específico da Educação em Astronomia [8]. Conforme [9], os temas de interesse que surgiram desde então são:

- Panorama da pesquisa sobre Educação em Astronomia;

- Estudos pilotos sobre aplicações de diferentes metodologias e técnicas de ensino de Astronomia na sala de aula;

- Envolvimento de instituições públicas no ensino de Astronomia no currículo escolar, tais como a NASA;

- Demonstrações, exercícios de laboratório e atividades de observação do céu real a olho nu e por telescópios, e do céu virtual em planetários;

- Ensino on-line de Astronomia e observações remotas por internet;

- Considerações sobre o uso da interdisciplinaridade da Astronomia nas aulas (arte, música, arqueoastronomia, teatro);

- Relações e aproximações entre as pseudociências e a Astronomia;

- Pesquisas sobre ensino e aprendizagem em Astronomia;

- Análise dos conteúdos de Astronomia nos livrostexto e seleção dos conteúdos mais significativos;

- Publicações que apresentam resultados de pesquisas sobre Educação em Astronomia;

- Discussões sobre cursos de Astronomia para professores;

- A importância dos observatórios e planetários para a Educação em Astronomia.

Parcerias entre a área de Educação e os astrônomos (profissionais e amadores) têm sido produtivas em vários países no sentido de promoverem mudanças no ensino quanto à Astronomia e na formação de professores da educação básica. Por exemplo, as ações articuladas entre sociedades científicas, grupos de pesquisa de universidades, astrônomos profissionais, astrônomos amadores, associações e clubes de Astronomia, exerceram profundas influências em órgãos governamentais ao ponto de produzirem amplas reformas curriculares e inserirem a Astronomia nas escolas, além de proporcionar a formação de professores. Isto ocorreu pelo menos nos seguintes países: Alemanha, França, Estados Unidos da América, Japão, Bulgária, México, Itália e Polônia [9]. 
No Brasil, talvez a ação mais exemplar e de relativo sucesso neste sentido tenha sido o Ano Internacional da Astronomia (IYA) em 2009. A resolução 62/200 da $62^{\text {a }}$ Assembleia Geral da ONU declarou 2009 como o Ano Internacional da Astronomia, gerando apoio financeiro a partir de órgãos públicos para projetos de divulgação científica na área de Astronomia e ciências afins, visando estimular a popularização da ciência e tecnologia e promover a melhoria da educação científica no País.

O edital MCT/SECIS/CNPq n.63/2008 foi uma demonstração de credibilidade nesta articulação Pro-Am-Es, pois proporcionou 2 milhões de reais para 75 projetos aprovados de ensino e divulgação em Astronomia, incluindo astrônomos amadores, profissionais e professores de escolas da educação básica, resultando em compras de telescópios automatizados com CCD para 80 IFETs (Institutos Federais de Educação Tecnológicas, hoje CEFETs), a edição de três livros amplamente distribuídos (Fascínio do Universo, Astronomia Hoje, Questões da OBA), distribuição de 35 mil DVDs com o documentário De Olho no Céu, ações e auxílios de eventos, tais como a Maratona da Via-Láctea, as 100 Horas de Astronomia, as Noites Galileanas, os encontros regionais de educação em astronomia (EREA) realizados em várias regiões do país, o primeiro Simpósio Nacional de Educação em Astronomia (SNEA), Semana Nacional de Ciência e Tecnologia (SNCT), reunião da Sociedade Brasileira para o Progresso da Ciência (SBPC), distribuição de 20.000 "galileoscópios" a escolas por todo o país, Olimpíada Brasileira de Astronomia e Astronáutica (OBA) e a produção de 250 kits e 140 mil folhetos da exposição Paisagens Cósmicas, abrangendo 725 lugares diferentes de todo o território nacional e visitados por 641.067 pessoas [10].

Em todo o mundo, foram 148 países participantes do IYA realizando mais de 50.000 eventos atendendo mais de 102 milhões de pessoas. Na ocasião, o Brasil foi um país que superou as expectativas internacionais, promovendo 16.369 eventos, dos quais participaram 2.292.675 pessoas, nos 201 estabelecimentos que reportaram suas atividades (foram 251 inicialmente registrados), sendo compostos de $25 \%$ de universidades e centros de pesquisas, $15 \%$ de planetários e museus de ciências e $60 \%$ de clubes e associações de astrônomos amadores, revelando a importante contribuição desta classe de amadores quanto à divulgação e popularização da Astronomia.

Como legado do IYA, criou-se em 2009 a Rede Brasileira de Astronomia (RBA), cuja missão nos anos seguintes seria dar prosseguimento às atividades semelhantes do IYA, unindo esforços entre profissionais, amadores e a educação formal e não formal. Contudo, a RBA desfaleceu-se em menos de um ano e as atividades nacionais de ensino e divulgação em Astronomia minguaram desde então. Por outro lado, a Rede de Astronomia Observacional (REA-Brasil) mantém suas atividades Pro-Am desde 1988, reunindo registros de astrônomos amadores em campanhas observacionais e remetendo os resultados a órgãos profissionais quando necessário.
De fato, os desafios e as possibilidades da Educação em Astronomia no Brasil continuam sendo muitos e a união de esforços entre os astrônomos profissionais, amadores e a escola (Pro-Am-Es) constitui-se ainda em um caminho potencialmente frutífero e pouco explorado. Em um levantamento bibliográfico apresentado por [11], é possível vislumbrar os desafios e possibilidades que a literatura da área apresenta para a Educação em Astronomia no Brasil:

\section{Desafios:}

- Lacunas na formação inicial de professores relativas a metodologias e conteúdos de ensino de Astronomia;

- Cursos de curta duração em Astronomia, normalmente denominados de "formação continuada", em geral, não promovem mudanças efetivas na prática docente;

- Carência de material bibliográfico e fonte segura de informações sobre Astronomia;

- Espetacularização da mídia e sensacionalismos exagerados sobre temas e fenômenos de Astronomia;

- Escassez de estabelecimentos dedicados à astronomia e pouco investimento na criação de espaços não formais de ensino (há poucos observatórios, planetários, associações, museus, etc);

- Potencial dos estabelecimentos (observatórios, planetários, associações e clubes) pouco aproveitado, com atividades pontuais e não generalizadas para o território nacional;

- Persistência de erros conceituais em livros didáticos e outros manuais didáticos;

- Quantidade reduzida de pesquisas sobre Educação em Astronomia (em espaços formais e não formais);

- Perda de valorização cultural e falta do hábito de olhar para o céu;

- Propagação em massa de concepções alternativas e mitos sobre fenômenos astronômicos;

- Falta de atualizações aos professores quanto a novas descobertas e informações sobre fenômenos astronômicos iminentes que poderiam ser aproveitados nas aulas;

- Subutilização da forte componente motivacional e interdisciplinar da Astronomia no ensino.

\section{Possibilidades:}

- Fomentar a criação, nas escolas, de Grupos de Estudos sobre Educação e Divulgação da Astronomia Interdisciplinar (Clubes de Astronomia);

- Reunir para o professor: fontes, conteúdos, propostas de atividades, sugestões metodológicas e didáticas, relatos;

- Estabelecer campanhas nacionais de observação do céu;

- Elaboração e envio de periódicos de Astronomia (boletins) que motivem professores e alunos a observar o céu; 
- Promover, durante congressos científicos, momentos de discussões com professores da educação básica, visando interações entre a comunidade de pesquisadores e os professores;

- Motivar órgãos de fomento para formação continuada em Astronomia para professores em parceria com universidades e astrônomos amadores;

- Aumentar a quantidade e a qualidade da produção de pesquisas sobre a Educação em Astronomia no Brasil;

- Utilizar metodologias de formação de professores que contribuam para a mudança da prática do professor no sentido de inserir astronomia em seu ensino;

- Massiva produção midiática nacional gratuita de documentários televisivos, publicações confiáveis e pôsteres didáticos de Astronomia;

- Desmistificar concepções e mitos em Astronomia por meio de ações nacionais e investimento em estabelecimentos não formais de ensino;

- Aproveitar nacionalmente o potencial dos estabelecimentos (observatórios, planetários, associações e clubes);

- Promover articulações nacionais entre as comunidades de astrônomos profissionais, amadores e as escolas.

\section{Considerações}

O Brasil possui potencial para desenvolver atividades de ensino e divulgação de Astronomia que atinjam simultaneamente todo o território nacional, como mostram estes exemplos de colaboração Pro-Am-Es. Se analisarmos brevemente o histórico da evolução do currículo escolar e da divulgação da Astronomia em alguns países, entenderemos a importância da união entre a escola e os astrônomos profissionais e amadores. Contudo, as pesquisas brasileiras sobre Educação em Astronomia repetidamente demonstram que foram poucas e lentas as mudanças efetivas para o ensino e divulgação da Astronomia em nosso país. Quando ocorrem, mostram-se pulverizadas como atividades localizadas, pontuais e rarefeitas em relação à extensão territorial do Brasil, embora tais ações localizadas regionalmente sejam louváveis. Acreditamos que já nos encontramos no tempo de superar estas atividades pontuais no sentido de promover articulações de âmbito coletivo visando atingir o território nacional como um todo. Temos potencial para isso, conforme apontado neste artigo e recomendamos a mobilização das comunidades de astrônomos profissionais, amadores, pesquisadores em ensino e os professores da educação básica a exercerem conjuntamente pressões em poderes públicos quanto a mudanças educacionais a favor do ensino e divulgação da Astronomia.

\section{Referências}

[1] D. Boyd, Journal of the British Astronomical Association 121, 2 (2011).

[2] R. Langhi e R. Nardi, Educação em Astronomia: Repensando a Formação de Professores (Escrituras, São Paulo, 2012), $215 \mathrm{p}$.

[3] R. Langhi e R. Nardi, Revista Brasileira de Ensino Física 31, 4 (2009).

[4] F. Braga-Ribas, B. Sicardy, J.L. Ortiz, C. Snodgrass, F. Roques, R. Vieira-Martins, J.I.B. Camargo, M. Assafin, R. Duffard, E. Jehin, et al., Nature 7494, 72 (2014).

[5] F. Braga-Ribas, Boletim da SAB (2014), disponível em http://www.sab-astro.org.br/noticias/1539415, acesso em out. 2015.

[6] ESO, Nota de Imprensa do ESO 1410 (2014), disponível em http://www.eso.org/public/brazil/news/ eso1410/, acesso em out. 2015.

[7] M. Gerbaldi, The Teaching of Astronomy (US Press, Cambridge, 1990).

[8] J. Pasachof and J. Percy (orgs), The Teaching of Astronomy (US Press, Cambridge, 1990).

[9] A. Fraknoi (ed), Cosmos in the Classroom 2007: Papers and Handouts from a Hands-on Symposium on Teaching Introductory Astronomy (ASP, California, 2007).

[10] T. Napoleão, in: X Encontro Regional de Educação em Astronomia, Campo Grande, 2011.

[11] R. Langhi e J. Vilaça, Revista Planetaria 7, 6 (2015). 